\title{
Model for the coherent optical manipulation of a single spin state in a charged quantum dot
}

\author{
G. Slavcheva* \\ Advanced Technology Institute, School of Electronics and Physical Sciences, University of Surrey, \\ Guildford GU2 7XH, Surrey, United Kingdom
}

(Received 28 December 2007; revised manuscript received 7 February 2008; published 25 March 2008)

\begin{abstract}
The optically driven coherent dynamics associated with the single-shot initialization and readout of a localized spin in a charged semiconductor quantum dot embedded in a realistic structure is theoretically studied using a new Maxwell-pseudospin model. Generalized pseudospin master equation is derived for description of the time evolution of spin coherences and spin populations in terms of the real state pseudospin (coherence) vector including dissipation in the system through spin-relaxation processes. The equation is solved in the time-domain self-consistently with the vector Maxwell equations for the optical wave propagation coupled to it via macroscopic medium polarization. Using the model, the long-lived electron spin coherence left behind a single resonant ultrashort optical excitation of the electron-trion transition in a charged quantum dot is simulated in the low- and high-intensity Rabi oscillation regime. Signatures of the polarized photoluminescence (PL) resulting from the numerical simulations, such as the appearance of a second echo pulse following the excitation and a characteristic nonmonotonic PL trace shape, specific for initial spin-up orientation, are discussed for realization of high-fidelity schemes for coherent readout of a single spin polarization state.
\end{abstract}

DOI: 10.1103/PhysRevB.77.115347

PACS number(s): 78.67.Hc, 37.10.Jk, 71.35.Pq, 78.20.Bh

\section{INTRODUCTION}

One of the challenges for the development of future technologies is the realization of next generation devices that control not only the electron charge, as in contemporary state-of-the-art electronics, but also its spin degree of freedom. Among the huge variety of proposed physical realizations of a solid-state qubit and respective schemes to realize quantum computing based on it, a single electron (hole) spin confined in a charged quantum dot $(\mathrm{QD})^{1,2}$ has recently attracted significant attention. This proposal is particularly promising due to a combination of advantages: It enables the implementation of ultrafast all-optically based quantum computation schemes through the optical spin orientation mechanism using circularly polarized ultrashort optical pulses, a technique of key importance for generation and manipulation of spin-polarized states in semiconductors. ${ }^{3}$ This, in turn, is a prerequisite for realization of quantum coherent control of the spin since a necessary condition for quantum coherence is the use of sufficiently short optical pulses so they can interact with the quantum system before it can be affected by its environment. On the other hand, QDs have the advantage of being intermediate between ordinary atoms and the higher-dimensional semiconductor structures characterized by complex many-body physics. Owing to the threedimensional-carrier localization and the discrete-level electronic structure, the electron spin confined in a QD is far less affected by the semiconductor environment, compared to the quantum wells and bulk, resulting in relatively long spincoherence times.

Spin states in QDs have been initially studied by measuring the average signal from a large ensemble of QDs. Long ensemble spin-coherence time $T_{2}^{*} \sim 100 \mathrm{~ns}$ in bulk semiconductors has been reported. ${ }^{4}$ Recent experiments on singleshot readout of an individual spin in GaAs and $\mathrm{In}(\mathrm{Ga}) \mathrm{As}$ single QDs have demonstrated long single electron spin energy relaxation times (spin-flip time) $T_{1}$ in the millisecond range (1-20 ms). ${ }^{5-7}$ Since $T_{2}$ may last as long as $2 T_{1},{ }^{8}$ long single spin-coherence times are anticipated. This has been recently confirmed by the extended single spin-coherence times $T_{2}>1 \mu \mathrm{s}$ found in a double QD in the high-intensity coherent regime ${ }^{9}$ employing the techniques of quantum coherent control, such as spin-echo pulse sequences, to suppress hyperfine-induced dephasing, due to interactions with the nuclear spins of the lattice ions. In this regime of resonant nonlinear coherent light-matter interactions, Rabi oscillations of the population between the discrete levels occur. In this respect, QDs are advantageous to the ordinary atoms since population flopping over many periods is possible for them, being systems with longer coherence times and larger dipole moments. Spin-decoherence times $T_{2}$ on the order of magnitude of $1-100 \mu \mathrm{s}$ (Refs. 10 and 11) are theoretically predicted. The long spin-relaxation and spin-decoherence times recently observed ensure a long-lived quantum state required for performing a large number of spin manipulations (quantum operations) during which coherence needs to be retained. On the other hand, owing to the angular momentum conservation, the polarization state of photons (the socalled flying qubit) can be converted into a localized spin (stationary qubit) and the process is reversible. This has direct implications for the transport of qubits from one location to another and allows for building up scalable architectures, in contrast to proposals based on conditional exciton dynamics (see, e.g., Ref. 12) which have problems with scalability.

One promising approach is to optically address individual carrier spins in semiconductor QDs and to manipulate them through optically excited states (charged excitons) by employing the techniques of coherent quantum control and optical orientation. Despite the recent progress achieved in coherent optical spin state preparation with near-unity fidelity, using laser cooling (optical pumping) techniques, ${ }^{13}$ single spin qubit detection in solid-state systems has only been achieved using transport measurements ${ }^{6,14}$ magnetic resonance force microscopy, ${ }^{15}$ and most recently off-resonant optical Faraday rotation, ${ }^{16}$ and the all-optical preparation, ma- 

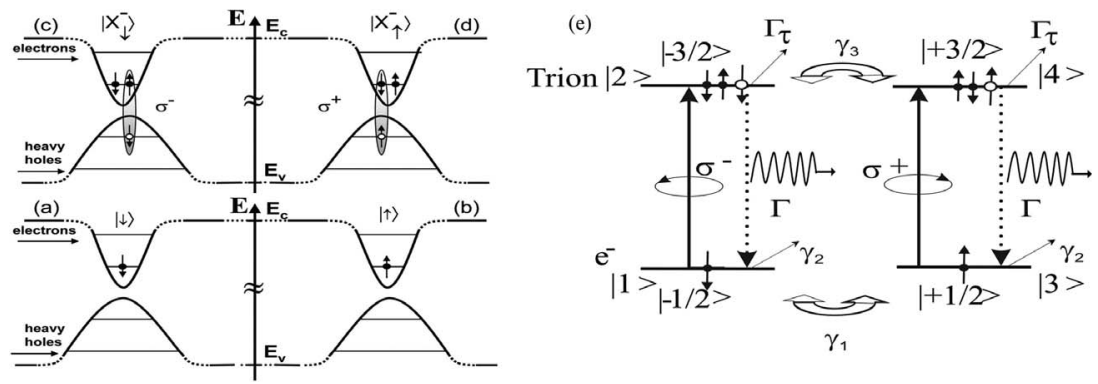

FIG. 1. Schematic representation of $[(a)$ and (b)] the initial electron states in a single singly charged quantum dot and the ground trion state created from it by (c) $\sigma^{-}$and (d) $\sigma^{+}$excitations of the heavy-hole excitonic transitions. (e) Energy-level diagram of a negatively charged exciton (trion) state in a single quantum dot. The levels are labeled by the total angular momentum projection along the propagation and quantization $z$ axis. Dipole-allowed optical transitions correspond to $\Delta J_{z}= \pm 1$ and the fundamental energy gap is $\hbar \omega_{0}$; coherent optical transitions excited by left $\left(\sigma^{-}\right)$- and right $\left(\sigma^{+}\right)$-circularly polarized light are designated by solid arrows, spontaneous optical transitions with rate $\Gamma$ (dashed arrows), transitions due to electron and hole-spin relaxation $\gamma_{1}$ and $\gamma_{3}$, respectively; spin-decoherence rates for electrons $\gamma_{2}$ and holes $\Gamma_{\tau}$.

nipulation, and detection still remains a challenging task.

A diversity of theoretical strategies based on charged excitons ${ }^{17}$ has been put forward using resonant or nonresonant optical excitation of a charged exciton (trion) with circularly polarized light. Schemes of how this trion permits the readout of a single electron spin during the spin-relaxation time have been discussed ${ }^{18,19}$ There is strong evidence from both recent experimental data, ${ }^{9,20}$ and theoretical computations ${ }^{18,19}$ that the intense resonant excitation of the trion transition suppresses the electron spin relaxation due to hyperfine interaction with nuclear spins of the lattice ions, which limits the ability to accurately measure the electron spin orientation at low temperatures. On the other hand, Rabi oscillations in QDs correspond to one-qubit rotation and therefore represent a step toward the implementation of quantum information processing in QDs. In this paper, we theoretically and numerically explore the possibility of exploiting low- and high-intensity ultrafast resonant coherent excitations for coherent optical initialization and detection of a single electron spin confined in a negatively charged QD embedded in realistic device geometries (e.g., nonlinear optical waveguides and semiconductor microcavities). This is achieved by developing a general theory and a numerical model, based on it, for rigorous description of circularly polarized ultrashort optical pulse interactions with the resonant nonlinearities in semiconductor QDs.

We shall study quantum dots charged with one single electron. Injection of a single electron into a quantum dot can be achieved by using, e.g., modulation doping in the barrier region adjusting the impurity doping level within the deltadoped layer to transfer on average one electron per dot and to populate only their lowest states, or by electrical injection. We focus on a model of lens-shaped quantum dots with lateral dimensions largely exceeding the height that is sufficiently general to represent a wide class of zero-dimensional systems (e.g., self-assembled InAs QDs grown by molecular beam epitaxy, GaAs natural islandlike QDs, formed by GaAs quantum well thickness fluctuations, or nanocrystal CdSe QDs). Due to the quasicylindrical symmetry of the QD about the quantization axis $z$, the single-particle states of the electrons in the conduction band and the holes in the valence band can be approximated by those of a pair of harmonic oscillators $^{21}$ [Figs. 1(a) and 1(b)].

The ground electron, heavy-hole, and light-hole states are degenerate with respect to their total angular momentum projections $\pm 1 / 2, \pm 3 / 2$, and $\pm 1 / 2$, respectively. We assume that the lowest heavy-hole state (with total angular momentum projection $\left.J_{z}= \pm 3 / 2\right)$ and light-hole state $\left(J_{z}= \pm 1 / 2\right)$ are split by an energy $\Delta_{h h-l h}$, and the band mixing of heavyhole and light-hole states is negligible. In what follows, we shall consider a resonant circularly polarized optical excitation that is restricted to the heavy-hole states only. The electromagnetic wave incident to the singly charged quantum dot is propagating along the quantization direction $z$ and is circularly polarized in a plane perpendicular to it. A single electron in the lowest orbital state is either in the spin-up or spin-down ground state [Figs. 1(a) and 1(b)]. The resonant circularly polarized optical excitation of the charged dot leads to the formation of a negatively charged exciton (trion) consisting of two electrons sitting at the same lowest (conduction band) electron quantum level, forming a spin singlet state, and a hole occupying the lowest valence band hole level. According to the exciton optical selection rules, which reflect the angular momentum conservation along the $z$ axis, $\sigma^{-}$-polarized light couples $|-3 / 2\rangle$ heavy-hole states and $|+1 / 2\rangle$ (spin-up) electron state with total angular momentum projection of -1 [Fig. 1(c)], whereas $\sigma^{+}$light couples $|+3 / 2\rangle$ heavy-hole state with $|-1 / 2\rangle$ electron state with total angular momentum projection of +1 [Fig. 1(d)]. It should be noted that the ground trion state optically created by adding $\sigma^{+}\left(\sigma^{-}\right)$exciton to the single electron in the dot can spontaneously decay only into the same initial electron state emitting circularly polarized light with the same polarization as the one of the stimulated resonant coherent excitation. Therefore, the polarization of the photoluminescence uniquely determines the initial electron spin projection $\left(\sigma^{+}\right.$polarized photoluminescence implies electron spin-up $|\uparrow\rangle$ projection, whereas $\sigma^{-}$polarized photoluminescence determines electron spin-down $|\downarrow\rangle$ projection). As has been pointed out, ${ }^{18}$ the selection rules permit many-cycle repetitions without loosing information about the electron spin state and the electron, and the trion spin-relaxation limits the measurement time of 
the photoluminescence with a given polarization. It is obvious that the longer the spin lifetime is, the better the chances to optically manipulate a particular spin state and to read out the state of a single electron spin during the spin-relaxation time are. Therefore, we shall be interested in the spin dynamics at low temperatures, since in this regime, the localized electron has a long spin-coherence time. It has been pointed out $^{11}$ that the most-likely dominant mechanism of electron spin relaxation in quantum dots at low temperatures is the hyperfine interaction with the frozen configuration of randomly oriented spins of the lattice nuclei. There are strong indications, however, that in the strong excitation regime, the electron spin relaxation in the nuclear field is suppressed by the intense resonant optical excitation through a mechanism similar to "motional" (dynamical) narrowing., 3

\section{THEORETICAL BACKGROUND}

In this paper, we investigate both the low- and the highintensity nonlinear excitation regimes. We have adopted the usual statistical interpretation of quantum mechanics that uses the ensemble point of view. In this view, the state vector or density matrix describes not a single system but an ensemble of identically prepared systems. Therefore, we shall model the resonant nonlinearity associated with the coherent optical trion transitions in a single charged quantum dot by an ensemble of multilevel systems (degenerate four-level systems [Fig. 1(e)] with density $N_{a}$ obtained by replication of the single-dot system. It should be noted that the individual members of the ensemble are independent: there is no interaction between the replicas within the ensemble and there is no transfer of population outside each replica, so that the conservation of the total occupation probability within each replica is preserved $\left(\rho_{11}+\rho_{22}+\rho_{33}+\rho_{44}=1\right)$ at all times. The initial assumption is that at $t=0$, we simultaneously create (by, e.g., optical pumping with circularly polarized light) electrons in the lowest orbital state, all having the same spin orientation. The systems have exactly the same occupation probability distributions under the driving laser field, so that all possible configurations (realizations) are going to be equivalent and the ensemble averaging procedure is straightforward. The justification of such an approach is based on the assumptions of validity of the ergodic hypothesis, namely, the equivalence of the time averages of an observable (in this case, the single-dot polarized photoluminescence, determined by the dynamics) and the ensemble average, which is the average at one time over a large number of systems, all of which have identical properties. In what follows, we apply proper normalization of the dot density in order to ensure a single dot within the simulation domain and show that there are strong indications that the ergodicity holds in the problem considered. Under these conditions, it has been demonstrated ${ }^{22}$ that quantum mechanics allows predictions of single systems based on macroscopic time averages of observables.

Therefore, we shall assume that the time dependence of the optically induced coherent spin generation and subsequent relaxation in a single quantum dot, averaged over a large number of successive measurements, is equivalent to the corresponding spin dynamics of an ensemble of identical quantum dots. The ensemble of homogeneously broadened degenerate four-level systems is resonantly coupled to an optical wave propagating along the $z$ direction, circularly polarized in a perpendicular plane to $z$. During its propagation, the optical wave interacts with the four-level medium schematically shown in Fig. 1, thereby inducing polarizations $P_{x}$ and $P_{y}$ along the $x$ and $y$ directions, respectively. The onedimensional Maxwell's curl equations in an isotropic medium read

$$
\begin{gathered}
\frac{\partial H_{x}(z, t)}{\partial t}=\frac{1}{\mu} \frac{\partial E_{y}(z, t)}{\partial z}, \\
\frac{\partial H_{y}(z, t)}{\partial t}=-\frac{1}{\mu} \frac{\partial E_{x}(z, t)}{\partial z},
\end{gathered}
$$

$$
\begin{gathered}
\frac{\partial E_{x}(z, t)}{\partial t}=-\frac{1}{\varepsilon} \frac{\partial H_{y}(z, t)}{\partial z}-\frac{1}{\varepsilon} \frac{\partial P_{x}(z, t)}{\partial t}, \\
\frac{\partial E_{y}(z, t)}{\partial t}=\frac{1}{\varepsilon} \frac{\partial H_{x}(z, t)}{\partial z}-\frac{1}{\varepsilon} \frac{\partial P_{y}(z, t)}{\partial t} .
\end{gathered}
$$

The time evolution of a discrete four-level system in an external time-dependent dipole-coupling perturbation is governed by the Liouville equation of motion for the complex density matrix $\hat{\rho}$,

$$
\frac{\partial \hat{\rho}}{\partial t}=\frac{i}{\hbar}[\hat{\rho}, \hat{H}]
$$

where $\hat{H}$ is the system Hamiltonian in the four-level basis [Fig. 1(e)], phenomenologically constructed assigning complex Rabi frequencies to the coherent optical dipole-allowed transitions in the quantum system. ${ }^{23,24}$ Since the initial electron spin can be either in spin-down state $|1\rangle$, or spin-up state $|3\rangle$, the corresponding Hamiltonian for each case is given by

$$
\begin{gathered}
\hat{H}_{1}=\hbar\left(\begin{array}{cccc}
0 & -\frac{1}{2}\left(\Omega_{x}-i \Omega_{y}\right) & 0 & 0 \\
-\frac{1}{2}\left(\Omega_{x}+i \Omega_{y}\right) & \omega_{0} & 0 & 0 \\
0 & 0 & 0 & 0 \\
0 & 0 & 0 & \omega_{0}
\end{array}\right), \\
\hat{H}_{2}=\hbar\left(\begin{array}{cccc}
0 & 0 & 0 & 0 \\
0 & \omega_{0} & 0 & 0 \\
0 & 0 & 0 & -\frac{1}{2}\left(\Omega_{x}+i \Omega_{y}\right) \\
0 & 0 & -\frac{1}{2}\left(\Omega_{x}-i \Omega_{y}\right) & \omega_{0}
\end{array}\right),
\end{gathered}
$$

where $\wp$ is the trion optical dipole transition matrix element.

The dynamics associated with the spin-flip (longitudinal) and spin-decoherence (transverse) relaxation processes and the trion radiative decay via spontaneous emission need to be taken into account in Eq. (2). Spin-flip relaxation of the electron spin due to hyperfine interaction with the lattice ion nuclear spin induces population transfer between the lowerlying levels, while the hole-spin relaxation in the trion state 

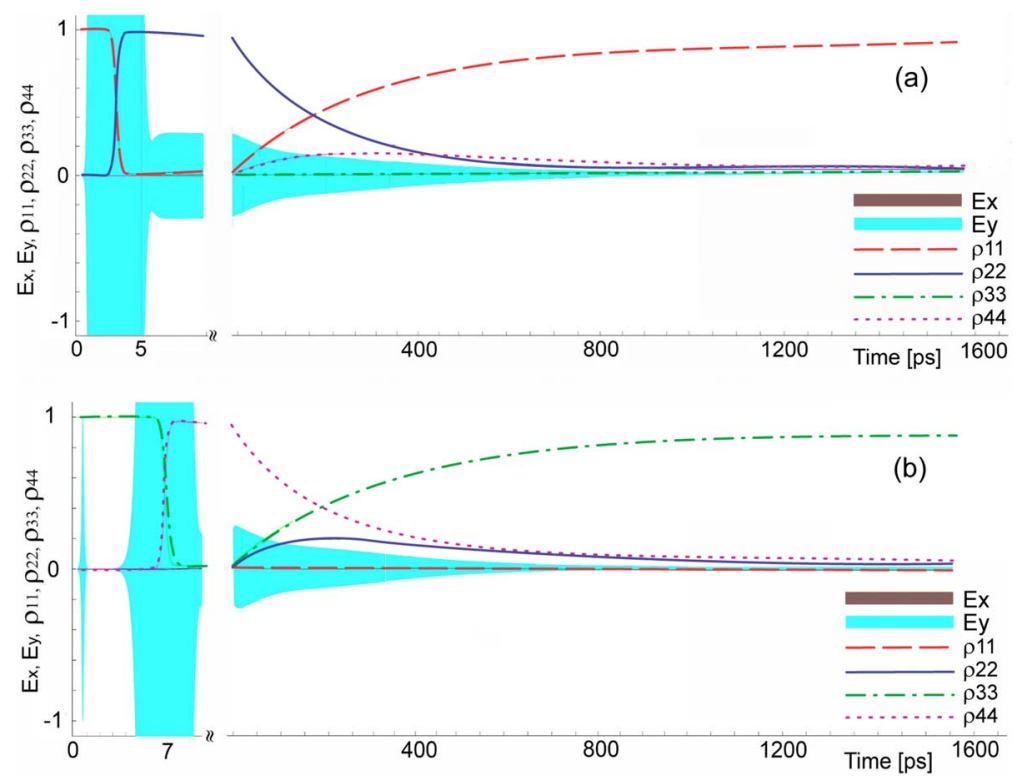

FIG. 2. (Color online) Time evolution of the $\sigma^{-}$-polarized optical pulse $\left(T_{p}=1.3 \mathrm{ps}\right.$, initial field amplitude $E_{0}=550 \mathrm{~V} \mathrm{~m}^{-1}$ ) $E$-field components and the corresponding spin population of all four states in Fig. 1(e); $\rho_{22}$ (blue curve, solid line) represents the trion $|-3 / 2\rangle$ state spin population $\left(\sigma^{-}\right.$-polarized photoluminescence) for (a) initially $|\downarrow\rangle$ populated state $|1\rangle$ [Fig. 1(e)]; (b) initial $|\uparrow\rangle$ state excited by a pulse with the same $\sigma^{-}$ helicity; (c) time-resolved $\sigma^{-}$-polarized photoluminescence of the trion $|-3 / 2\rangle$ state for $|\downarrow\rangle$ (blue curve, solid line) and $|\uparrow\rangle$ (red curve, dashed line) initial electron spin states.

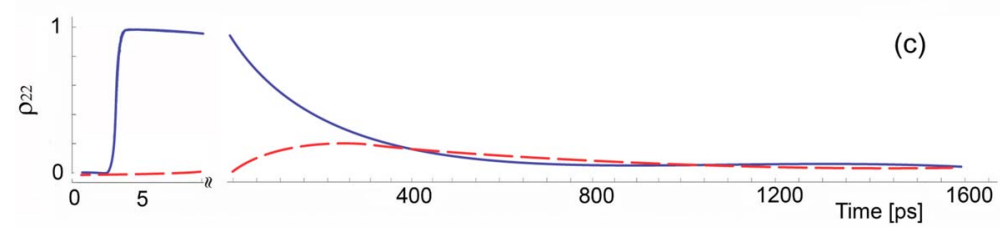

through phonon-assisted processes induces population transfer between the upper-lying levels [Fig. 1(e)]. ${ }^{18}$ For the sake of generality, we shall denote the electron spin-down population transfer rate via spin-flip processes from level $|1\rangle$ to level $|3\rangle$ by $\gamma_{13}$ and the opposite transition spin-up population transfer rate by $\gamma_{31}$; similarly, the trion state hole-spin population transfer rates between levels $|2\rangle$ and $|4\rangle$ will be denoted by $\gamma_{24}$ and $\gamma_{42}$, respectively. Electron spin decoherence, trion spin decoherence, and spontaneous emission rate are denoted by $\gamma_{2}, \Gamma_{\tau}$, and $\Gamma$, respectively. Using these notations, the following generalized master equation for the density matrix $\hat{\rho}$ can be derived, based on rate equation considerations:

$$
\frac{\partial \hat{\rho}}{\partial t}=\frac{i}{\hbar}[\hat{\rho}, \hat{H}]+\hat{\sigma}-\hat{\Gamma}_{t} \hat{\rho},
$$

where we have introduced $\hat{\sigma}$ matrix accounting for the spin population transfer, according to

$$
\hat{\sigma}=\left(\begin{array}{cccc}
\operatorname{Tr}\left(\hat{\Gamma}_{1} \hat{\rho}\right) & 0 & 0 & 0 \\
0 & \operatorname{Tr}\left(\hat{\Gamma}_{2} \hat{\rho}\right) & 0 & 0 \\
0 & 0 & \operatorname{Tr}\left(\hat{\Gamma}_{3} \hat{\rho}\right) & 0 \\
0 & 0 & 0 & \operatorname{Tr}\left(\hat{\Gamma}_{4} \hat{\rho}\right)
\end{array}\right),
$$

defining the matrices,

$$
\begin{aligned}
& \Gamma_{1}=\left(\begin{array}{cccc}
-\gamma_{13} & 0 & 0 & 0 \\
0 & \Gamma & 0 & 0 \\
0 & 0 & \gamma_{31} & 0 \\
0 & 0 & 0 & 0
\end{array}\right), \quad \Gamma_{2}=\left(\begin{array}{cccc}
0 & 0 & 0 & 0 \\
0 & -\Gamma-\gamma_{24} & 0 & 0 \\
0 & 0 & 0 & 0 \\
0 & 0 & 0 & \gamma_{42}
\end{array}\right), \\
& \Gamma_{3}=\left(\begin{array}{cccc}
\gamma_{13} & 0 & 0 & 0 \\
0 & 0 & 0 & 0 \\
0 & 0 & -\gamma_{31} & 0 \\
0 & 0 & 0 & \Gamma
\end{array}\right), \quad \Gamma_{4}=\left(\begin{array}{cccc}
0 & 0 & 0 & 0 \\
0 & \gamma_{24} & 0 & 0 \\
0 & 0 & 0 & 0 \\
0 & 0 & 0 & -\Gamma-\gamma_{42}
\end{array}\right),
\end{aligned}
$$

and the spin-decoherence matrix $\hat{\Gamma}_{t}$ accounts for the dissipation in the system due to loss of spin coherence,

$$
\hat{\Gamma}_{t}=\left(\begin{array}{cccc}
0 & \Gamma_{\tau} & \gamma_{2} & \gamma_{2} \\
\Gamma_{\tau} & 0 & \Gamma_{\tau} & \Gamma_{\tau} \\
\gamma_{2} & \Gamma_{\tau} & 0 & \Gamma_{\tau} \\
\gamma_{2} & \Gamma_{\tau} & \Gamma_{\tau} & 0
\end{array}\right) .
$$

Note that due to symmetry relationships $\left(\rho_{i j}=\rho_{j i}^{*}\right)$, there are only ten independent components of the density matrix.

Employing the generalized pseudospin formalism based on the commutator Lie algebra of $\mathrm{SU}(\mathrm{N})$ group ${ }^{25,26}$ expanding the density matrix and the system Hamiltonian of a discrete four-level quantum system in terms of the $\lambda$ generators of SU(4) algebra, a pseudospin master equation for the real 15-dimensional state vector is derived, governing the stimulated dynamics, spin-flip relaxation, and spin decoherence, 


$$
\frac{\partial S_{j}}{\partial t}= \begin{cases}f_{j k l} \Gamma_{k} S_{l}+\frac{1}{2} \operatorname{Tr}\left(\hat{\sigma} \hat{\lambda}_{j}\right)-\frac{1}{T_{j}}\left(S_{j}-S_{j e}\right), & j=1,2, \ldots, 12, \\ f_{j k l} \Gamma_{k} S_{l}+\frac{1}{2} \operatorname{Tr}\left(\hat{\sigma} \hat{\lambda}_{j}\right), & j=13,14,15,\end{cases}
$$

where $\boldsymbol{\Gamma}$ is the torque vector, $f$ is the fully antisymmetric tensor of the structure constants of SU(4) group, and $T_{j}$ are the phenomenologically introduced nonuniform spindecoherence times describing the relaxation of the real state vector components $S_{j}$ toward their equilibrium values $S_{j e}$. The $\lambda$ generators of SU(4) algebra are calculated using the definitions given in Ref. 25, and the matrix $\sigma$ in Eq. (8) is the diagonal matrix from Eq. (5), expressed in terms of the real state vector components.

Expressions for the longitudinal spin population relaxation times $T_{13}, T_{14}$, and $T_{15}$ due to both spin-relaxation processes and spontaneous emission have been derived through the second term in Eq. (8), giving

$$
\begin{gathered}
T_{13}=\frac{4}{2 \Gamma+\gamma_{13}+\gamma_{24}}, \quad T_{14}=\frac{12}{3 \gamma_{13}+\gamma_{24}+6 \gamma_{31}}, \\
T_{15}=\frac{6}{\gamma_{24}+3\left(\Gamma+\gamma_{42}\right)} .
\end{gathered}
$$

From the equations of motion for the off-diagonal density matrix components, using the relationship between the real state vector components and the density matrix components, the following spin-decoherence times are obtained: namely, $T_{1}=T_{2}=T_{5}=T_{6}=T_{7}=T_{8}=T_{11}=T_{12}=1 / \Gamma_{\tau}$ and $T_{3}=T_{4}=T_{9}$ $=T_{10}=1 / \gamma_{2}$.

The torque vector $\boldsymbol{\Gamma}$ components are expressed in terms of the $\lambda$ generators of the SU(4) Lie algebra according to Ref. 25. Using Hamiltonian (3), the torque vector is calculated for the two initial spin orientations considered, giving correspondingly

$$
\begin{aligned}
& \boldsymbol{\Gamma}_{1}=\left(-\Omega_{x}, 0,0,0,0,0,-\Omega_{y}, 0,0,0,0,0, \omega_{o},-\frac{\omega_{o}}{\sqrt{3}}, \sqrt{\frac{2}{3}} \omega_{o}\right), \\
& \boldsymbol{\Gamma}_{2}=\left(0,0,0,0,0,-\Omega_{x}, 0,0,0,0,0,-\Omega_{y}, \omega_{o},-\frac{\omega_{o}}{\sqrt{3}}, \sqrt{\frac{2}{3}} \omega_{o}\right) .
\end{aligned}
$$

Substituting back in Eq. (8) taking into account all nonvanishing components of the $f$ tensor, a system describing the time evolution of the 15-dimensional state vector is obtained, which in matrix form reads,

$$
\frac{\partial \mathbf{S}}{\partial t}=\left\{\begin{array}{l}
\hat{M} \mathbf{S}+\frac{1}{2} \operatorname{Tr}(\hat{\sigma} \hat{\lambda})-\operatorname{diag}\left(1 / T_{1}, 1 / T_{2}, \ldots, 1 / T_{12}\right)\left(\mathbf{S}-\mathbf{S}_{E}^{*}\right), \\
\hat{M} \mathbf{S}+\frac{1}{2} \operatorname{Tr}(\hat{\sigma} \hat{\lambda}),
\end{array}\right.
$$

where $S_{E}^{*}=\left(S_{1 e}, S_{2 e}, S_{3 e}, \ldots, S_{12 e}\right)$ denotes a subset of the equilibrium vector $S_{E}=\left(0,0,0, \ldots, S_{13 e}, S_{14 e}, S_{15 e}\right)$ responsible for the spin decoherence. Note that the first 12 components of $S_{E}$ vanish because of the incoherent nature of the energy input that maintains the system at a definite level of excitation $^{27}$ and only the population term components are nonzero. In the above equation, $\mathbf{M}$ is $15 \times 15$ antisymmetric block matrix with 13 independent components given by

$$
\hat{M}=\left(\begin{array}{ccc}
\underline{P}_{6 \times 6} & \underline{Q}_{6 \times 6} & \underline{R}_{6 \times 3} \\
-\underline{Q}_{6 \times 6}^{T} & \underline{P}_{6 \times 6} & \underline{S}_{6 \times 3} \\
-\underline{R}_{3 \times 6}^{T} & -\underline{S}_{3 \times 6}^{T} & \underline{0}_{3 \times 3}
\end{array}\right),
$$

and the block matrices have the following explicit form given for initial spin-down populated level (the derivation for initial spin-up populated level is similar):

$$
\begin{aligned}
& \hat{P}=\left(\begin{array}{cccccc}
0 & 0 & 0 & 0 & 0 & 0 \\
0 & 0 & -\frac{\Omega_{y}}{2} & 0 & 0 & 0 \\
0 & \frac{\Omega_{y}}{2} & 0 & 0 & 0 & 0 \\
0 & 0 & 0 & 0 & \frac{\Omega_{y}}{2} & 0 \\
0 & 0 & 0 & -\frac{\Omega_{y}}{2} & 0 & 0 \\
0 & 0 & 0 & 0 & 0 & 0
\end{array}\right), \\
& \hat{Q}=\left(\begin{array}{cccccc}
\omega_{0} & 0 & 0 & 0 & 0 & 0 \\
0 & -\omega_{0} & \frac{\Omega_{x}}{2} & 0 & 0 & 0 \\
0 & \frac{\Omega_{x}}{2} & 0 & 0 & 0 & 0 \\
0 & 0 & 0 & \omega_{0} & \frac{\Omega_{x}}{2} & 0 \\
0 & 0 & 0 & \frac{\Omega_{x}}{2} & 0 & 0 \\
0 & 0 & 0 & 0 & 0 & \omega_{0}
\end{array}\right), \\
& \hat{R}=\left(\begin{array}{ccc}
\Omega_{y} & 0 & 0 \\
0 & 0 & 0 \\
0 & 0 & 0 \\
0 & 0 & 0 \\
0 & 0 & 0 \\
0 & 0 & 0
\end{array}\right), \quad \hat{S}=\left(\begin{array}{ccc}
-\Omega_{x} & 0 & 0 \\
0 & 0 & 0 \\
0 & 0 & 0 \\
0 & 0 & 0 \\
0 & 0 & 0 \\
0 & 0 & 0
\end{array}\right) \text {. }
\end{aligned}
$$


For the special case of a two-level system, the Hamiltonian is $2 \times 2$ and the $\lambda$ generators of the SU(2) Lie algebra are simply the Pauli matrices. In this case, the pseudospin system is reduced to the one derived by Feynman et al. ${ }^{28}$ During its propagation in the resonantly absorbing or amplifying medium, the polarized optical pulse induces polarization along $x$ and $y$ axes, perpendicular to the propagation direction. The macroscopic medium polarization is given by

$$
\mathbf{P}=-e N_{a} \operatorname{Tr}(\hat{\rho} \cdot \hat{Q}),
$$

where $N_{a}$ is the density of the ensemble of resonantly absorbing or amplifying four-level systems. Taking into account the form of interaction Hamiltonian (3), expanding the local displacement operator $\hat{Q}$ components and the density matrix in terms of $\lambda$ generators of SU(4) Lie algebra, ${ }^{26}$ the Cartesian polarization components are expressed in terms of the real pseudospin (state) vector components,

$$
\sigma^{-}\left\{\begin{array}{l}
E_{x}(z=0, t)=E_{o} \operatorname{sech}(10 \Gamma) \cos \left(\omega_{o} t\right) \\
E_{y}(z=0, t)=-E_{0} \operatorname{sech}(10 \Gamma) \sin \left(\omega_{o} t\right),
\end{array}\right.
$$

where $E_{0}$ is the initial field amplitude, $\Gamma=\left[t-\left(T_{p}\right)\right] /\left(T_{p} / 2\right)$, and $T_{p}$ is the pulse duration.

\section{NUMERICAL SIMULATIONS}

The system under investigation is a GaAs/AlGaAs selfassembled modulation-doped molecular-beam-epitaxygrown QD with $5 \mathrm{~nm}$ height (see, e.g., Ref. 31) sandwiched between two $50 \mathrm{~nm} \mathrm{Al}_{0.3} \mathrm{Ga}_{0.7} \mathrm{As}$ barriers with refractive indices $n_{\mathrm{GaAs}}=3.63$ and $n_{\mathrm{AlGaAs}}=3.46$ at the trion transition resonance wavelength $(\lambda=787 \mathrm{~nm})$. The circularly polarized pulse center frequency $\omega_{0}=2.39 \times 10^{15} \mathrm{rad} \mathrm{s}^{-1}$ is tuned in resonance with the energy splitting between the ground electron and the singlet trion states of $1.58 \mathrm{eV},{ }^{31}$ corresponding to a wavelength $\lambda=787 \mathrm{~nm}$, and the pulse shape is given by a hyperbolic secant envelope [Eq. (18)] with duration $T_{p}$ $=1.3 \mathrm{ps}$. Throughout the simulations, the initial field amplitude is varied from a low field $E_{0}=550 \mathrm{~V} \mathrm{~m}^{-1}$, through $E_{0}$ $=3 \times 10^{6} \mathrm{~V} \mathrm{~m}^{-1}$, representing a $\pi$ pulse, completely inverting the spin population, to $E_{0}=4 \times 10^{7} \mathrm{~V} \mathrm{~m}^{-1}$, corresponding to an $\sim 12 \pi$ pulse, inducing six full Rabi flops. An estimate for the trion dipole moment is obtained using the exciton Bohr radius, giving a value of $\wp=4.8 \times 10^{-28} \mathrm{C} \mathrm{m}$. The active simulation domain is $5 \mathrm{~nm}$ long, corresponding to a typical volume of a cylindrical quantum dot (with diameter $d=10 \mathrm{~nm}$ and height $h=5 \mathrm{~nm})$ of $3.93 \times 10^{-25} \mathrm{~m}^{-3}$. The quantum dot (resonant four-level system) density $N_{a}=2.5$ $\times 10^{24} \mathrm{~m}^{-3}$ is selected to give on average one dot within this microscopic volume. Therefore, although the volume density is a very large number, the simulations are restricted to this particular spatial domain which contains only a single dot.

We shall be interested in the low-temperature regime. Transitions between the lower-lying initial electron levels oc-

$$
P_{x}=-\wp N_{a} S_{1}, \quad P_{y}=-\wp N_{a} S_{7} .
$$

The above polarizations act as source terms in the vector Maxwell's equation for the optical wave propagation [Eq. (1)].

The Maxwell curl equations [Eq. (1)] are solved selfconsistently with the master pseudospin equations [Eq. (12)], using Eq. (17), for the fields and the real-vector components in the time domain employing the finite-difference timedomain (FDTD) technique ${ }^{29}$ without invoking any approximations, such as slowly varying wave approximation and rotating-wave approximation.

The initial boundary value problem requires the knowledge of the whole time history of the initial field along some characteristic, e.g., at $z=0$ (the left boundary of our simulation domain). The circularly polarized optical pulse is modeled by two orthogonal linearly polarized optical waves, phase shifted by $\pi / 2,{ }^{23,24,30}$

$\sigma^{+}\left\{\begin{array}{l}E_{x}(z=0, t)=E_{o} \operatorname{sech}(10 \Gamma) \cos \left(\omega_{o} t\right) \\ E_{y}(z=0, t)=E_{0} \operatorname{sech}(10 \Gamma) \sin \left(\omega_{o} t\right),\end{array}\right.$

cur due to the hyperfine interaction of the electron spin with the frozen random configuration of the nuclear spins of the lattice ions. ${ }^{11,18}$ In what follows, we shall assume that the electron spin population transfer rates between the lowerlying energy levels are equal, namely, $\gamma_{13}=\gamma_{31}=\gamma_{1}$, and the hole-spin population transfer rates between the upper-lying levels are also equal, namely, $\gamma_{24}=\gamma_{42}=\gamma_{3}$.

Throughout the simulations, the following parameters are kept constant: the electron spin-relaxation rate due to electron spin precession in the frozen random configuration of lattice ion nuclear spins is set to $\gamma_{1}=500 \mathrm{ps}^{-1}$ as a lower limit of the theoretical rates calculated in Refs. 10 and 11. Transitions between the upper-lying levels also occur due to the hole-spin relaxation in the trion state which is a phononassisted process and yields relaxation rates that can be comparable to or even longer than those for electrons $\mathrm{s}^{32-34}$ with relaxation rate $\gamma_{3}=170 \mathrm{ps}^{-1}$ in agreement with Ref. 20. The trion radiative decay (recombination) rate is set to $\Gamma$ $=400 \mathrm{ps}^{-1}$ as inferred from time-resolved photoluminescence. ${ }^{20}$ The electron spin-decoherence (transverse) rate is taken to be $\gamma_{2}=450 \mathrm{ps}^{-1}$ (see, e.g., Ref. 35), and the trion state spin-decoherence rate is assumed to be $\Gamma_{\tau} \sim 2 \gamma_{3}$ $=340 \mathrm{ps}^{-1}$.

We shall be interested in the long-lived spin coherence left behind an ultrashort pulse when the excitation intensity is varied. The time evolution of the left-circularly polarized pulse Cartesian components $E_{x}$ and $E_{y}$ and the spin population of all four levels at a point within the QD is given in Fig. 2 at the initial field amplitude $E_{0}=550 \mathrm{~V} \mathrm{~m}^{-1}$.

The initial spin orientation is assumed to be spin-down (state $|1\rangle)$, taking the initial state of the system to be its equilibrium state [Fig. 2(a)]. The left-circularly $\left(\sigma^{-}\right)$polarized ultrashort optical pulse initially slowly excites the spin 

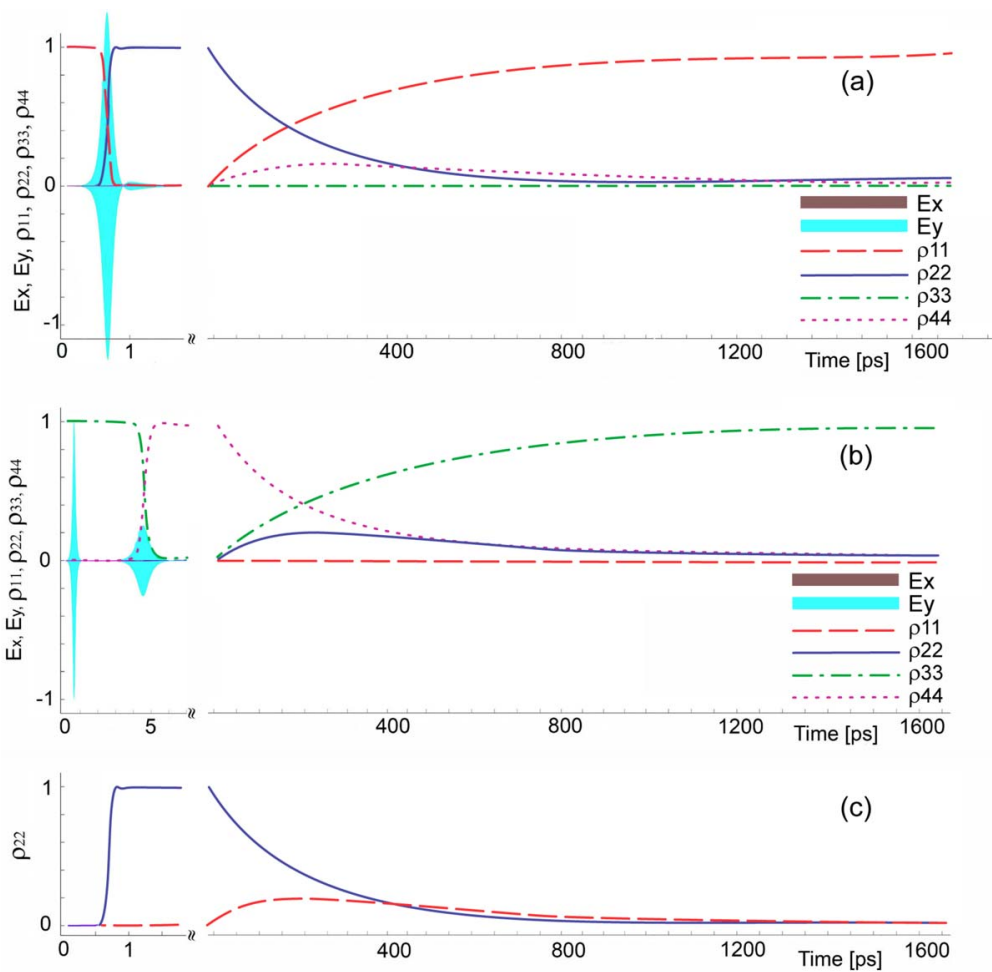

FIG. 3. (Color online) Time evolution of the $\sigma^{-}$-polarized optical pulse $\left(T_{p}=1.3 \mathrm{ps}\right.$, initial field amplitude $E_{0}=3 \times 10^{6} \mathrm{~V} \mathrm{~m}^{-1}$, giving a pulse area of $\pi$ ) $E$-field components and the corresponding spin population of all four states in Fig. $1(\mathrm{e}) ; \rho_{22}$ (blue curve, solid line) represents the trion $|-3 / 2\rangle$ state spin population $\left(\sigma^{-}\right.$-polarized photoluminescence) for (a) initially $|\downarrow\rangle$ populated state $|1\rangle$ [Fig. 1(e)]; (b) initial $|\uparrow\rangle$ state excited by a pulse with the same $\sigma^{-}$helicity; (c) timeresolved $\sigma^{-}$-polarized photoluminescence of the trion $|-3 / 2\rangle$ state for both cases (a) and (b). population in agreement with the optical dipole selection rules. As the time elapses, the propagating resonant pulse experiences amplification in the QD medium and the amplified pulse nearly completely excites the spin population into the upper level $|2\rangle$. Note that the pulse split up is due to the transfer of spin population between the lower- and upperlying levels, which creates polarization density causing reemission back to the field. The evolution of the population $\rho_{22}$ of state $|2\rangle$ (blue curve, solid line) describes the spin population of $|-3 / 2\rangle$ trion state excited by $\sigma^{-}$-polarized pulse, and therefore represents a measure of the intensity of the $\sigma^{-}$-polarized photoluminescence, since the rate of the $\sigma^{-}$-photon emission is $\Gamma \rho_{22}{ }^{18}$ The long-time dynamics develops at a time scale much longer than the pulse duration due to the long spin-relaxation times involving spin population transfer between the spin-down and spin-up states.

If the initial spin population resides in $|3\rangle$ with spin-up orientation, the optical pulse with the same $\sigma^{-}$polarization does not affect the second system $(|3\rangle \rightarrow|4\rangle)$ [Fig. 2(b), initial section of the green curve (dashed-dotted line) representing $\left.\rho_{33}\right]$ and the spin population of the $|-3 / 2\rangle$ trion state remains low [Fig. 2(b), blue curve (solid line)], slowly varying in time due solely to transitions between levels $|1\rangle$ $\rightarrow|3\rangle,|2\rangle \rightarrow|4\rangle$ and spontaneous emission. Note that at the simulation time $t=7 \mathrm{ps}$ [Fig. 2(b)], the spin population of the state $|3\rangle$ is exactly equal to the spin population of level $|4\rangle$, and therefore a dipole is formed due to the spin-relaxation processes. The dipole acts as a source in Maxwell's equations leading to an additional electric field pulse which appears at later times following the excitation. The time traces of the $\sigma^{-}$-polarized photoluminescence for the two cases considered above is plotted on the same plot in Fig. 2(c) showing that a sufficiently long time interval exists
( $\sim 400 \mathrm{ps})$ within which a differentiation between the two initial spin states can be made with great fidelity, thereby allowing for uniquely detecting the spin polarization through time-dependent polarized photoluminescence experiments. Note that the $\sigma^{-}$-polarized PL decay for spin-down and spin-up initial states is considerably different. While the polarized PL for initial spin-down state exhibits exponential decay [Fig. 2(c), blue curve, solid line], the PL for initial spin-up state is a nonmonotonic function of time with characteristic rising time, reaching a maximum and a subsequent decay [Fig. 2(c), red curve, dashed line]. This opens up the possibility of exploiting the time-resolved polarized PL characteristic shape in the time domain for high-fidelity determination of the initial spin state. On the other hand, the appearance of a second pulse after the initial excitation in the case of initial spin-up state could be used as a probe for differentiation between the two initial spin states thus determining the initial spin-up orientation with high accuracy.

In our simulations, we pay special attention to the optical excitations with pulse area of $\pi$ (or odd multiples of $\pi$ ), since it completely inverts the population in a two-level system. In Fig. 3(a), the coherent time evolution of the $E$-field components and the corresponding spin populations of the four levels after a passage of an ultrashort $\pi$ pulse is plotted. At this relatively high intensity, the field amplitude exhibits saturation although has not reached the stationary value. The pulse is split into two and its trailing edge is due to the population transferred to the second two-level system $(|3\rangle$ $|4\rangle)$. The spin population $\rho_{22}$ of level $|2\rangle$ [blue curve, solid line in Fig. 3(a)] represents the $\sigma^{-}$-photoluminescence decay. If the initial spin population is in spin-up state residing in state $|3\rangle$, a $\sigma^{-} \pi$ pulse initially does not affect the $(|3\rangle-|4\rangle)$ system until sufficiently large population is transferred to the 

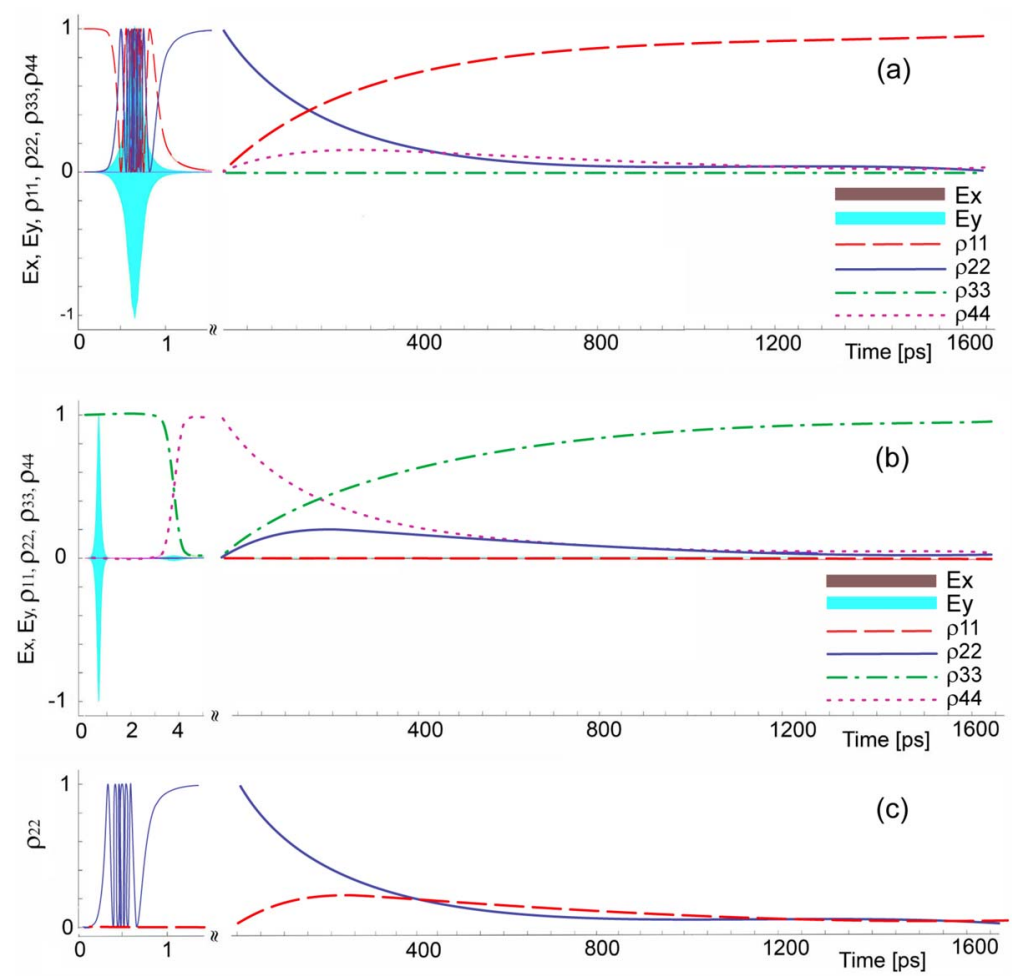

FIG. 4. (Color online) Time evolution of the $\sigma^{-}$-polarized optical pulse $\left(T_{p}=1.3 \mathrm{ps}\right.$, initial field amplitude $E_{0}=4 \times 10^{7} \mathrm{~V} \mathrm{~m}^{-1}$, giving a pulse area of $12 \pi$ ) $E$-field components and the corresponding spin population of all four states in Fig. $1(\mathrm{e}) ; \rho_{22}$ (blue curve, solid line) represents the trion $|-3 / 2\rangle$ state spin population $\left(\sigma^{-}\right.$-polarized photoluminescence) for (a) initially $|\downarrow\rangle$ populated state $|1\rangle$ [Fig. 1(e)]; (b) initial $|\uparrow\rangle$ state excited by a pulse with the same $\sigma^{-}$helicity; (c) timeresolved $\sigma^{-}$-polarized photoluminescence of the trion $|-3 / 2\rangle$ state for both cases (a) and (b). first two-level system $(|1\rangle-|2\rangle)$ and a dipole is created which emits radiation back to the field [Fig. 3(b)]. This, in turn, leads to the appearance of a second pulse at later times. The second pulse experimental detection would provide means to distinguish between the two initial spin states with great accuracy. A comparison of the photoluminescence traces for both initial spin orientation cases is plotted in Fig. 3(c) showing relatively long-time interval of $(\sim 400 \mathrm{ps})$ within which the initial spin state can be detected with great accuracy after which the two states become indistinguishable.

In the high excitation regime, the time evolution of the left-circularly polarized pulse Cartesian components $E_{x}$ and $E_{y}$ and the spin population of all four levels at a point within the QD is given in Fig. 4 at the initial field amplitude $E_{0}$ $=4 \times 10^{7} \mathrm{~V} \mathrm{~m}^{-1}$, corresponding to an even multiples of $\pi$ pulse $(12 \pi$ pulse). At this high excitation intensity, the optical field amplitude saturates and the short-time dynamics exhibits Rabi oscillations of the spin population. The number of the full Rabi flops is determined by the pulse area $12 \pi$ yielding six full Rabi flops [Fig. 4(a)]. It becomes apparent that the Rabi oscillations over the width of the pulse suppress the spin relaxation, leading to a slightly longer coherence decay time (in the case of a single ultrashort pulse) in agreement with Ref. 18. It is clear that the longer the pulse duration is, the more extended in time the suppression of the time decay of the coherence is. Figure 4(b) shows the corresponding time traces when the initial electron spin state is $|\uparrow\rangle$. In accordance with the optical selection rules, the spin population $\rho_{33}$ residing in state $|3\rangle$ remains unaffected by the propagating pulse at short times. However, at longer times $(\sim 4 \mathrm{ps})$, the spin populations $\left(\rho_{33}\right.$ and $\left.\rho_{44}\right)$ equalize, leading to the formation of an electric dipole between the states, which, in turn, emits back radiation to the field, thus creating an after pulse, similar to the previous case (with much lower amplitude). The two time traces of the photoluminescence of the trion $|-3 / 2\rangle$ state are plotted in Fig. 4(c) showing a long enough time interval ( $\sim 400 \mathrm{ps})$ within which differentiation between the two electron spin states can be made. As in the previous two cases considered, the characteristic shape of the polarized PL allows for differentiation between the two initial spin orientations with great accuracy.

In Fig. 5, the time trace of the right-circularly polarized electric field $\sigma^{+}$components and the spin population of the levels is shown for the case of initial spin-up population

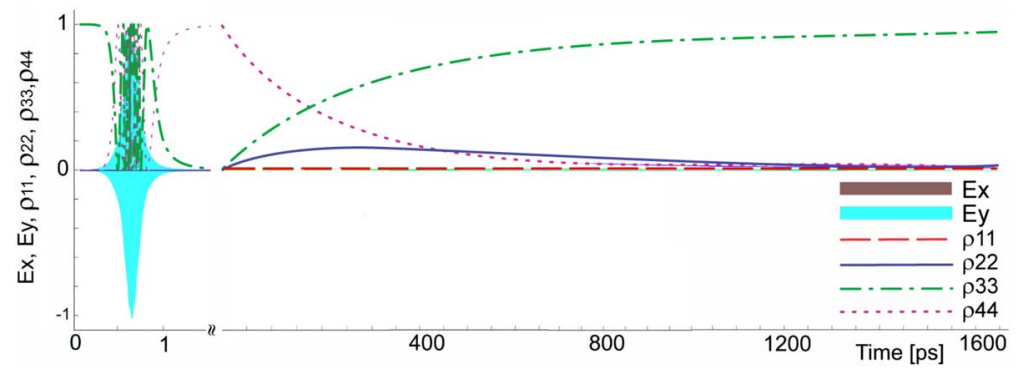

FIG. 5. (Color online) Time evolution of the $\sigma^{+}$-polarized optical pulse $\left(T_{p}=1.3 \mathrm{ps}\right.$, initial field amplitude $E_{0}=4 \times 10^{7} \mathrm{~V} \mathrm{~m}^{-1}$ ) $E$-field components and the corresponding spin population of all four states in Fig. 1(e); $\rho_{44}$ (magenta curve, dotted line) represents the trion $|+3 / 2\rangle$ state spin population $\left(\sigma^{+}\right.$-polarized photoluminescence) for initially $|\uparrow\rangle$ populated state $|3\rangle$ [Fig. 1(e)]. 


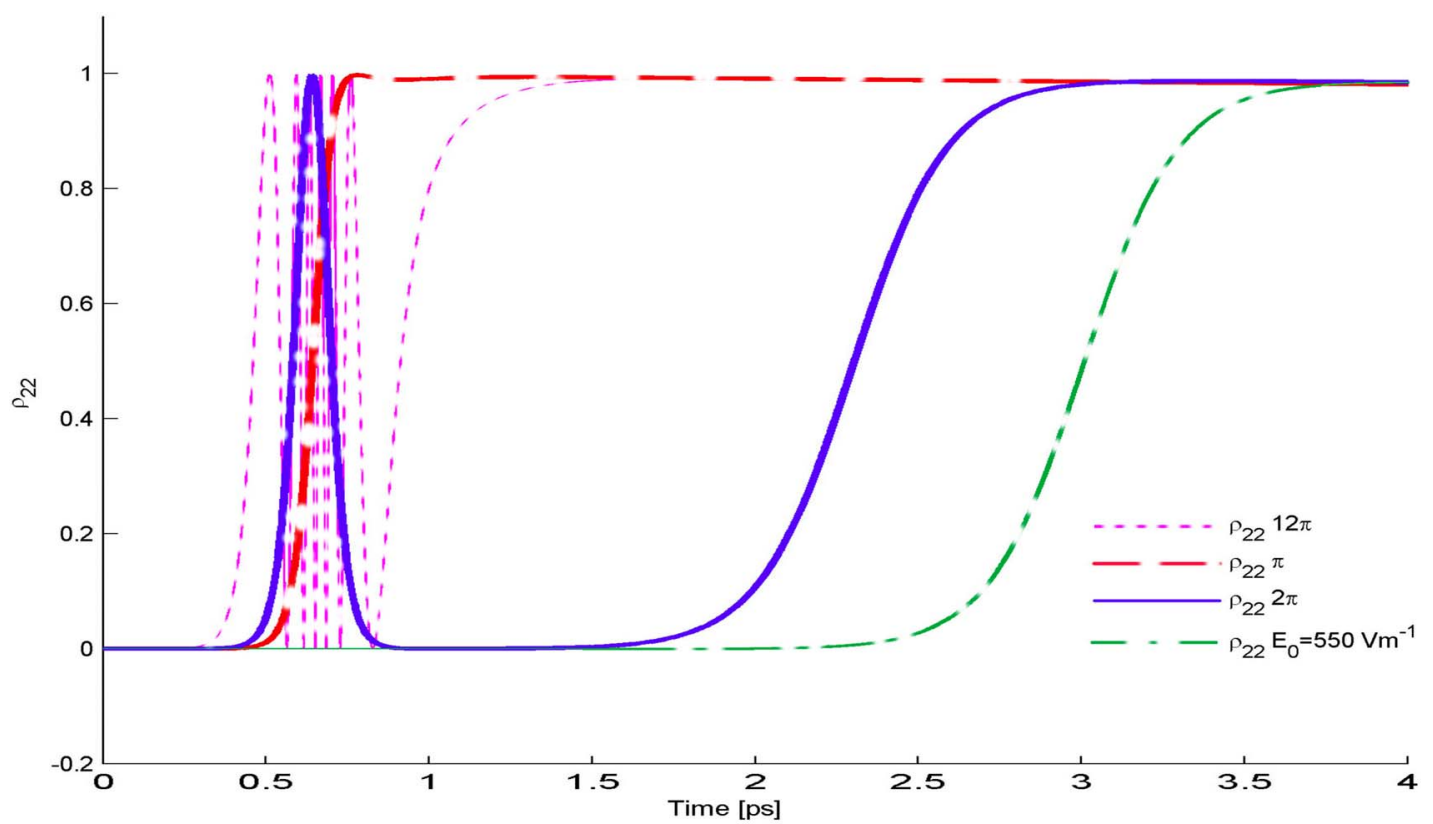

FIG. 6. (Color online) Short-time dynamics of the $\sigma^{-}$-polarized PL $\left(\rho_{22}\right)$ induced by an optical pulse with pulse duration $T_{p}=1.3$ ps for initial field amplitude $E_{0}=550 \mathrm{~V} \mathrm{~m}^{-1}$ (green, dashed-dotted line), $E_{0}=3 \times 10^{6} \mathrm{~V} \mathrm{~m}^{-1}$, corresponding to a pulse area $\theta=\pi$ (red, long-dashed line), $E_{0}=6.68673 \times 10^{6} \mathrm{~V} \mathrm{~m}^{-1}, \theta=2 \pi$ (blue, solid line), and $E_{0}=4 \times 10^{7} \mathrm{~V} \mathrm{~m}^{-1}, \theta=12 \pi$ yielding six full Rabi flops of the spin population (magenta, short-dashed line).

residing in level $|3\rangle$. The simulation is performed keeping all parameters the same as in the previous simulations. Comparison with Fig. 4 shows that the population of the trion $|3 / 2\rangle$ spin state $\rho_{44}$ exhibits Rabi flops similar to the ones in Fig. 4 and its time decay represents the $\sigma^{+}$-polarized photoluminescence. In this case, the second system is excited by the right-circularly polarized pulse (in agreement with the dipole optical selection rules) and the first system is only affected through the spin-flip and trion spontaneous decay processes.

Comparison of the short-time dynamics of the polarized PL following an ultrashort $\sigma^{-}$-pulse excitation of initial spindown state for the three cases considered above is given in Fig. 6. It shows decrease in the initial rising times with increasing the pulse area (pulse intensity). For the special case of a $2 \pi$ pulse excitation, the spin population of level $|2\rangle$ performs a full Rabi flop (blue curve, solid line).

The goal of maximizing the difference between the PL intensity for the two initial spin orientation cases naturally invokes the idea of undoing (time reversing) the time evolution of the spin coherence using techniques similar to spinecho pulse sequences. However, we note that those kinds of techniques are not applicable for undoing the spin population evolution since a population transfer is involved between the two two-level systems rather than solely spin-decoherence processes. These techniques would be useful, however, for rephasing of the pseudospin vector transverse dephasing.

Finally, turning to the model justification, besides the proper normalization of the dot density aiming to ensure a single dot in the microscopic simulation domain, the validity of the ergodic hypothesis and hence of our approach is reinforced by the following conditions: (i) the system of simultaneous equations is linear and the time integration is per- formed up to very long times, when the system has approached dynamical equilibrium; (ii) the simulated dynamics of a laser-driven dot plus radiation field system is Markovian since the dynamics at any future time moment is solely calculated from the previous time moment (by virtue of the FDTD method of calculation); iii) the density matrix relaxes to a stationary value which does not depend on the initial conditions. ${ }^{18}$ In fact, these are all well-known conditions under which the ergodicity is valid, although a rigorous mathematical proof still does not exist.

\section{CONCLUSIONS}

In conclusion, we have presented a dynamical model for the coherent spin dynamics induced by an ultrashort optical excitation through optical orientation mechanism. The adopted approach models the coherent light-matter interaction exploiting the $\mathrm{SU}(\mathrm{N})$ Lie group symmetries in a discrete multilevel quantum system (particularly adapted to the QD description) and the full vector treatment of the electromagnetic wave propagation, thereby accounting for the electromagnetic field polarization. The model has been applied to the ultrafast spatiotemporal dynamics involved in the trion state of a singly charged QD. Selective generation of specific spin states by circularly polarized light with predefined helicity and subsequent detection through the polarized timeresolved photoluminescence have been demonstrated. The simulations imply two distinct ways of reliable coherent initial spin state detection, namely, through the pulse echo appearing at later times following the initial excitation, specific only to initial state with spin-up orientation, and through the 
shape of the polarized PL trace in time showing a maximum again for this initial state in contrast to the single-exponential decay characterizing the spin-down initial state. The simulations show the onset of the high-intensity optical Rabi oscillation regime suppressing the spin-relaxation processes. The role of the effects such as inhomogeneous broadening ${ }^{36}$ and pure dephasing ${ }^{37}$ in a more realistic case of ensemble of QDs will be discussed in a separate paper within the framework of the present formalism.

\section{ACKNOWLEDGMENTS}

I would like to thank V. Kolev for valuable discussions. Support from the EPSRC under Grant No. EP/D060958/1 is gratefully acknowledged. *g.slavcheva@surrey.ac.uk

${ }^{1}$ D. Loss and D. P. DiVincenzo, Phys. Rev. A 57, 120 (1998).

${ }^{2}$ Semiconductor Spintronics and Quantum Computation, edited by D. D. Awschalom, D. Loss, and N. Samarth (Springer-Verlag, Heidelberg, 2002).

${ }^{3}$ Optical Orientation, edited by B. Meier and B. P. Zakharchenya (North-Holland, Amsterdam, 1984).

${ }^{4}$ J. M. Kikkawa, I. P. Smorchkova, N. Samarth, and D. D. Awschalom, Science 227, 1284 (1997); J. M. Kikkawa and D. D. Awschalom, Phys. Rev. Lett. 80, 4313 (1998); J. A. Gupta, D. D. Awschalom, X. Peng, and A. P. Alivisatos, Phys. Rev. B 59, R10421 (1999).

${ }^{5}$ A. C. Johnson, J. R. Petta, J. M. Taylor, A. Yacoby, M. D. Lukin, C. M. Marcus, M. P. Hanson, and A. C. Gossard, Nature (London) 435, 925 (2005).

${ }^{6}$ J. M. Elzerman, R. Hanson, L. H. Willems van Beveren, B. Witkamp, L. M. K. Vandersypen, and L. P. Kouwenhoven, Nature (London) 430, 431 (2004).

${ }^{7}$ M. Kroutvar, Y. Ducommun, D. Heiss, M. Bichler, D. Schuh, G. Abstreiter, and J. Finley, Nature (London) 432, 81 (2004).

${ }^{8}$ W. A. Coish and D. Loss, Phys. Rev. B 70, 195340 (2004).

${ }^{9}$ J. R. Petta, A. C. Johnson, J. M. Taylor, E. A. Laird, A. Yacoby, M. D. Lukin, C. M. Marcus, M. P. Hanson, and A. C. Gossard, Science 309, 2180 (2005).

${ }^{10}$ A. V. Khaetskii, D. Loss, and L. Glazman, Phys. Rev. Lett. 88, 186802 (2002); R. de Sousa and S. Das Sarma, Phys. Rev. B 67, 033301 (2003)

${ }^{11}$ I. A. Merkulov, Al. L. Efros, and M. Rosen, Phys. Rev. B 65, 205309 (2002).

${ }^{12}$ X. Li, Y. Wu, D. Steel, D. Gammon, T. H. Stievater, D. S. Katzer, D. Park, C. Piermarocchi, and L. J. Sham, Science 301, 809 (2003).

${ }^{13}$ M. Atatüre, J. Dreiser, A. Badolato, A. Högele, K. Karrai, and A. Imamoglu, Science 312, 551 (2006).

${ }^{14}$ F. H. L. Koppens, C. Bulzert, K. J. Tielrooij, I. T. Vink, K. C. Nowack, T. Meunier, L. P. Kouwenhoven, and L. M. K. Vandersypen, Nature (London) 442, 766 (2006); M. Xiao, I. Martin, E. Yablonivich, and H. W. Jiang, ibid. 430, 435 (2004).

${ }^{15}$ D. Rugar, R. Budakian, H. J. Mamin, and B. W. Chui, Nature (London) 430, 329 (2004).

${ }^{16}$ M. Atatüre, J. Dreiser, A. Badolato, and A. Imamoglu, Nat.
Phys. 3, 101 (2007).

${ }^{17}$ S. Cortez, O. Krebs, S. Laurent, M. Senes, X. Marie, P. Voisin, R. Ferreira, G. Bastard, J.-M. Gérard, and T. Amand, Phys. Rev. Lett. 89, 207401 (2002); E. Pazy, E. Biolatti, T. Calarco, I. D’Amico, P. Zanardi, F. Rossi, and P. Zoller, Europhys. Lett. 62, 175 (2003)

${ }^{18}$ A. Shabaev, Al. L. Efros, D. Gammon, and I. A. Merkulov, Phys. Rev. B 68, 201305(R) (2003).

${ }^{19}$ P. Chen, C. Piermarocchi, L. J. Sham, D. Gammon, and D. G. Steel, Phys. Rev. B 69, 075320 (2004).

${ }^{20}$ A. Greilich, R. Oulton, E. A. Zhukov, I. A. Yugova, D. R. Yakovlev, M. Bayer, A. Shabaev, Al. L. Efros, I. A. Merkulov, V. Stavarache, D. Reuter, and A. Wieck, Phys. Rev. Lett. 96, 227401 (2006).

${ }^{21}$ A. Wojs, P. Hawrylak, S. Fafard, and L. Jacak, Phys. Rev. B 54, 5604 (1996).

${ }^{22}$ G. Hegerfeld, Fortschr. Phys. 46, 595 (1998).

${ }^{23}$ G. Slavcheva and O. Hess, Phys. Status Solidi C 3, 2414 (2006).

${ }^{24}$ G. Slavcheva and O. Hess, Optical and Quantum Electronics (Springer, Berlin, 2006), Vol. 38, p. 973.

${ }^{25}$ F. T. Hioe and J. H. Eberly, Phys. Rev. Lett. 47, 838 (1981).

${ }^{26}$ F. T. Hioe, Phys. Rev. A 28, 879 (1983).

${ }^{27}$ P. K. Aravind, J. Opt. Soc. Am. B 3, 1025 (1986).

${ }^{28}$ R. P. Feynman, F. L. Vernon, and R. W. Hellwarth, J. Appl. Phys. 28, 49 (1957).

${ }^{29}$ A. Taflove, Computational Electrodynamics: The Finite-Difference Time-Domain Method (Artech, Northwood, MA, 1995).

${ }^{30}$ G. Slavcheva and O. Hess, Phys. Rev. A 72, 053804 (2005).

${ }^{31}$ A. Hartmann, Y. Ducommun, E. Kapon, U. Hohenester, and E. Molinari, Phys. Rev. Lett. 84, 5648 (2000).

${ }^{32}$ D. V. Bulaev and D. Loss, Phys. Rev. Lett. 95, 076805 (2005).

${ }^{33}$ T. Takagahara, Phys. Rev. B 62, 16840 (2000).

${ }^{34}$ T. Flissikowski, I. A. Akimov, A. Hundt, and F. Henneberger, Phys. Rev. B 68, 161309(R) (2003).

${ }^{35}$ S. E. Economou, R.-B. Liu, L. J. Sham, and D. G. Steel, Phys. Rev. B 71, 195327 (2005).

${ }^{36}$ S. Schneider, P. Borri, W. Langbein, U. Woggon, J. Förstner, A. Knorr, R. L. Sellin, D. Ouyang, and D. Bimberg, Appl. Phys. Lett. 83, 3668 (2003).

${ }^{37}$ B. Krummheuer, V. M. Axt, T. Kuhn, I. D’Amico, and F. Rossi, Phys. Rev. B 71, 235329 (2005). 